\title{
Optical Heterodyne Micro-vibration Detection based on All-fiber Acousto-optic Superlattice Modulation
}

\author{
Wending Zhang, Zhen Chen, Biqiang Jiang, Ligang Huang, Dong Mao, Feng Gao, Ting Mei, Dexing \\ Yang, Lin Zhang, and Jianlin Zhao
}

\begin{abstract}
We propose a configuration of optical heterodyne micro-vibration detection based on an all-fiber acousto-optic superlattice modulation structure that acts as both frequency shifter and reflector, simultaneously. The vibration information within the frequency range between $1 \mathrm{~Hz}$ to $150 \mathrm{kHz}$ of a piezoelectric mirror (PZM) has been experimentally measured by using this all-fiber optical heterodyne detection configuration. The minimal measurable vibration amplitude and the resolution are around $0.013 \mathrm{~nm}$ and $10 \mathrm{pm}$ in the region of tens to hundreds of kilohertz, respectively. The configuration not only has advantages of compact size, easy alignment and non-contact measurement, but also gains high accuracy, which provides a promising alternative and could be applied in the compact and portable instruments based on optical heterodyne detection.
\end{abstract}

Index Terms-optical heterodyne detection, acousto-optic superlattice modulation, acousto-optic frequency shifter, micro-vibration.

\section{INTRODUCTION}

$\mathrm{O}$ PTICAL heterodyne detection is one of the main methods of optical coherent detection. Due to its advantages in high sensitivity, accuracy and stronger anti-interference ability, optical heterodyne detection has been widely used in fields of linewidth measurement for the ultra-narrow linewidth laser [1], detection of the

Manuscript received NOV. 28, 2016. This work is financially supported by National Natural Science Foundation of China (NSFC) (11404263, 61675169, 11634010, 61377055, 61405161, and 11574161), Fundamental Research Funds for the Central Universities (3102017zy021), and Natural Science Foundation of Shaanxi Province (S2015YFJQ0805).

Wending Zhang, Zhen Chen, Biqiang Jiang, Dong Mao, Ting Mei, Dexing Yang, and Jianlin Zhao are with Key Laboratory of Space Applied Physics and Chemistry, Ministry of Education; and Shaanxi Key Laboratory of Optical Information Technology, School of Science, Northwestern Polytechnical University, Xi'an 710072, China (e-mail: zhangwd@nwpu.edu.cn; zhenc@mail.nwpu.edu.cn; bqjiang@nwpu.edu.cn; maodong@nwpu.edu.cn; ting.mei@ieee.org; dxyang@nwpu.edu.cn,jlzhao@nwpu.edu.cn).

Biqiang Jiang, and Lin Zhang are with Aston Institute of Photonic Technologies, Aston University, Birmingham B4 7ET, UK (e-mail: bqjiang@nwpu.edu.cn; 1.zhang@aston.ac.uk).

Ligang Huang is with Key Laboratory of Optoelectronic Technology \& Systems (Ministry of Education), Chongqing University, Chongqing 400044, China (e-mail: lghuang@cqu.edu.cn).

Feng Gao is with MOE Key Laboratory of Weak-Light Nonlinear Photonics, TEDA Applied Physics Institute and School of Physics, Nankai University, Tianjin 300457, China (e-mail: fenggao@nankai.edu.cn). nanoparticles [2], measurement for material's thermal conductivity [3], micro/nano structure imaging [4], etc. Furthermore, it is worth noting that optical heterodyne detection also has a wide application in the micro-vibration sensing, such as precision mechanism monitoring [5], hydrophone [6], etc.

The key part of an optical heterodyne detection configuration is a frequency shifter to generate a frequency-shifted light and then form a carrier signal with high signal-to-noise ratio (SNR). To date, many optical elements, such as bulk-optic Bragg cells and integrated optical waveguides [7-10], have been used as frequency shifters. However, these optical elements need precise optical alignment. An all-fiber frequency shifter can avoid such difficulty naturally. Note that the first all-fiber acousto-optic frequency shifter was constructed by using a dynamic grating introduced by the acoustic flexural wave in a two-mode fiber (TMF) in 1986 [11, 12], and has been used to construct fiber-optic Fabry-Perot interferometers [13]. In 2015, we have also constructed an all-fiber optical heterodyne detection configuration based on an all-fiber acousto-optic structure [14], which acted as both the frequency shifter and the coupler at the same time. This configuration has high measurement accuracy and adjustable SNR in measurement of actual objects. However, the separation of the frequency-shifted light needed the use of the evanescent wave coupling technique, leading to decrease stability of the device.

In this paper, we propose and demonstrate an all-fiber optical heterodyne configuration based on an all-fiber acousto-optic superlattice modulation (AOSLM) structure for micro-vibration detection. The AOSLM structure plays dual roles of the frequency shifter and the reflector. In this experiment, the vibration waveform within the frequency range between $1 \mathrm{~Hz}$ to $150 \mathrm{kHz}$ of a piezoelectric mirror (PZM) has been measured by using this all-fiber optical heterodyne detection configuration. The minimal measurable vibration amplitude and resolution are around $0.013 \mathrm{~nm}$ and $10 \mathrm{pm}$ in the region of tens to hundreds of kilohertz, respectively.

\section{THEORETICAL ANALYSIS}

When an acoustic longitudinal wave (ALW) propagates along an unjacketed fiber Bragg grating (FBG), the FBG will be perturbed with a spatial sinusoidal phase modulation based on the elasto-optical effect [15-20]. The periodic modulation of the dielectric constant can produce a sequence of sidebands in spatial frequency with the formation as [15] 


$$
\begin{aligned}
\frac{\varepsilon(z)-n_{0}^{2}}{M n_{0}^{2}} & =J_{0}(a) \cos K z+\sum_{n=1}^{\infty} J_{n}(a)\left\{( - 1 ) ^ { n } \operatorname { c o s } \left[\left(K+n k_{s}\right) z\right.\right. \\
& \left.\left.-n \Omega_{s} t\right]+\cos \left[\left(K-n k_{s}\right) z+n \Omega_{s} t\right]\right\},
\end{aligned}
$$

where $M$ is the modulation depth of the dielectric constant, $n_{0}$ is the refractive index of the fiber core, $a=K s_{0} / k_{\mathrm{s}}, K$ is the wave vector of the FBG, $s_{0}$ is the peak strain produced by the ALW, $k_{\mathrm{s}}$ and $\Omega_{\mathrm{s}}$ are the wave vector and the angular frequency of the ALW, respectively. According to Eq. (1), note that a sequence of ghosts of the FBG are formed at spatial frequencies given by successive spatial sidebands of $K$. In other words, the ALW produces a series of traveling gratings and hence generates a series of sidebands on both sides of the reflection spectrum of the FBG. The reflection coefficient of the $\pm n$-order sidebands can be given by [21]

$$
R_{ \pm n}=\frac{\kappa_{ \pm n}^{2} \sinh ^{2}\left(\gamma_{ \pm n} L\right)}{\Delta \beta_{ \pm n} \sinh ^{2}\left(\gamma_{ \pm n} L\right)+\cosh ^{2}\left(\gamma_{ \pm n} L\right)},
$$

where $\gamma_{ \pm n}=\sqrt{\kappa_{ \pm n}^{2}-\Delta \beta_{ \pm n}^{2}}, \kappa_{ \pm n}$ is the coupling coefficient of the $\pm n$-order sideband, $\Delta \beta_{ \pm n}=2 \pi n_{0}\left(\lambda^{-1}-\lambda_{0}^{-1}\right) \pm n \pi / \lambda_{s}$ is the detuning from the central wavelength of $\pm n$-order sideband, $\lambda_{0}$ is the resonance wavelength of the FBG, $\lambda$ is the wavelength of the probe light, $\lambda_{\mathrm{s}}$ is the wavelength of the ALW, and $L$ is the length of the FBG. The resonance wavelength of the $\pm n$-order sideband can be expressed as [21]

$$
\lambda_{ \pm n}=\lambda_{0} \pm \frac{n \lambda_{0}^{2} f_{s}}{2 n_{0} v_{s}},
$$

where $f_{\mathrm{s}}$ and $v_{\mathrm{s}}=5760 \mathrm{~m} / \mathrm{s}$ are the frequency and the phase velocity of the ALW, respectively. The positive sideband numbers correspond to the long resonance wavelengths, vice versa. Meanwhile, the frequency of the light reflected by the $\pm n$-order sideband is shifted by $\mp n f_{\mathrm{s}}$ with respect to the probe light frequency when the propagation of the probe light is opposite to that of the ALW [22, 23]. Therefore, the AOSLM structure can act as both the frequency shifter and the coupler, simultaneously, and be used to construct the all-fiber optical heterodyne detection configuration.

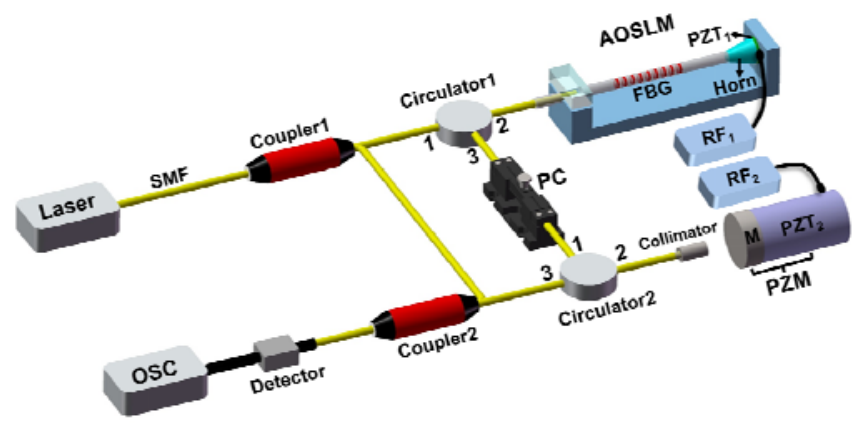

FIG. 1. Experimental configuration of the all-fiber optical heterodyne micro-vibration detection based on the AOSLM structure. SMF: single-mode fiber; FBG: fiber Bragg grating; RF: radio frequency signal; PC: polarization controller; M: mirror; PZM: piezoelectric mirror; OSC: oscilloscope.

Figure 1 is the experimental configuration of the all-fiber optical heterodyne micro-vibration measurement based on the AOSLM structure. With $\mathrm{RF}_{1}$ applied on the $\mathrm{PZT}_{1}$, an $\mathrm{ALW}$ is generated and then magnified at the tip of the horn acoustic transducer and propagates along the unjacketed FBG, resulting in an AOSLM structure. The beam from a tunable laser (Tunics, T100S-CL-WB) is divided into two paths by coupler 1 . One of the beam enters the AOSLM structure via circulator 1 , and then is reflected by the $+n$-order sideband of the AOSLM structure. Meanwhile, the frequency of the reflected beam is downshifted from that of the probe light by an amount equal to $-n f_{\mathrm{s}}$ due to opposite propagation directions between the probe light and the ALW [23]. Subsequently, the reflected beam with frequency of $f-n f_{\mathrm{s}}$ is sent to the collimator via circulator 2 to collect the vibration information of the PZM, which is constructed by attaching a gilded mirror $(\mathrm{M})$ on the surface of a cylindrical piezoelectric transducer $\left(\mathrm{PZT}_{2}\right)$ driven by $\mathrm{RF}_{2}$. Both the reflected light from port 3 of circulator 2 and the beam without frequency shifting from coupler 1 are combined via coupler 2 . Thus, the combined beams generate a light beat, which is recorded in an oscilloscope (OSC) with a detector.

Without $\mathrm{RF}_{2}$ applied on the PZM, the light beat used as carrier signal can be expressed as $S=\beta \mathrm{AB} \cos \left(2 \pi n f_{\mathrm{s}} \mathrm{t}\right)$, where $\beta$ is the conversion coefficient of the detector, and $\mathrm{A}$ and $\mathrm{B}$ are amplitudes of two beams, respectively. With a change in optical path of the frequency-shifted light produced by the vibration $u(t)$ of PZM, the vibration information is loaded on the carrier signal, and then the phase modulation signal is generated, which can be expressed as $S=\beta A B \cos \left[2 \pi n f_{\mathrm{s}} \mathrm{t}+4 \pi u(\mathrm{t}) / \lambda\right]$. Because the complex vibration can be written as the sum of its Fourier components, a simple vibration $u(t)=U \cos \left(2 \pi f_{0} t\right)$ with only one Fourier component is considered with $U$ and $f_{0}$ being the amplitude and the frequency of the vibration, respectively. The phase modulation signal can produce a sequence of frequency sidebands whose amplitudes are given by a standard Bessel function expansion [14]

$$
\begin{array}{r}
S=\beta A B\left\{J_{0}\left(\frac{4 \pi U}{\lambda}\right) \cos \left(2 \pi n f_{s} t\right)+\sum_{m=1}^{\infty} J_{m}\left(\frac{4 \pi U}{\lambda}\right),\right. \\
\left.\left[\cos \left(\left(n f_{s}+m f_{0}\right) t\right)+(-1)^{m} \cos \left(\left(n f_{s}-m f_{0}\right) t\right)\right]\right\}
\end{array}
$$

and the corresponding Fourier spectrum of Eq. (4) can be expressed as

$$
\begin{aligned}
F(f) & =\frac{\beta A B}{2}\left\{J_{0}\left(\frac{4 \pi U}{\lambda}\right) \delta\left(f-n f_{s}\right)+\sum_{m=1}^{\infty} J_{m}\left(\frac{4 \pi U}{\lambda}\right)\right. \\
& {\left.\left[(-1)^{m} \delta\left(f-\left(n f_{s}-m f_{0}\right)\right)+\delta\left(f-\left(n f_{s}+m f_{0}\right)\right)\right]\right\} }
\end{aligned} .
$$

According to Eq. (5), it can be known that the Fourier spectrum is composed of a series of frequency components. By demodulating the Fourier spectrum of the phase modulation signal, the vibration information of the PZM can be obtained, including the amplitude and the period.

\section{RESULTS AND DICUSSIONS}

Now we construct the experimental configuration of the all-fiber optical heterodyne micro-vibration detection based on the AOSLM structure. Firstly, the AOSLM structure composed of a FBG and an acoustic transducer is constructed. The acoustic transducer is the same as that of our previous works $[24,25]$. An axial mode $\mathrm{PZT}_{1}$ with a resonance frequency of $1.5 \mathrm{MHz}$ is attached to an aluminum horn, and the other side is mounted on a steel plate. The resonance 
wavelength and reflection efficiency of the FBG are $1550.403 \mathrm{~nm}$ and $22 \mathrm{~dB}$, respectively, as shown the black curve in Fig. 2(a). The grating length of the FBG is $50 \mathrm{~mm}$, and the fiber diameter was etched to 30 $\mu \mathrm{m}$ with hydrofluoric (HF) acid to increase the acousto-optic coupling efficiency [26, 27]. The tip of the aluminum horn was reduced to the diameter same as that of the FBG and subsequently glued to the FBG with UV epoxy.

By illuminating the FBG with a broadband light source and detecting the reflected spectrum using an optical spectrum analyzer (OSA), the reflection properties of the AOSLM can be measured. As shown in Fig. 2(a), the black curve is the reflection spectrum of FBG without $\mathrm{RF}_{1}$ applied on the $\mathrm{PZT}_{1}$, and the red curve is the reflection spectrum of the AOSLM structure by turning on the $\mathrm{RF}_{1}$ with $f_{\mathrm{s}}=1.5 \mathrm{MHz}$ and $\mathrm{V}_{\mathrm{pp}}=10 \mathrm{~V}$. The resonance wavelength of the \pm 1 -order reflection peaks are $\lambda_{-1}=1550.133 \mathrm{~nm}$ and $\lambda_{+1}=1550.747 \mathrm{~nm}$, respectively. In this experiment, the +1 -order sideband was selected as the reflected peak with a downshifted frequency equaling to the ALW frequency $\mathrm{f}_{\mathrm{a}}=1.5 \mathrm{MHz}$ [23].
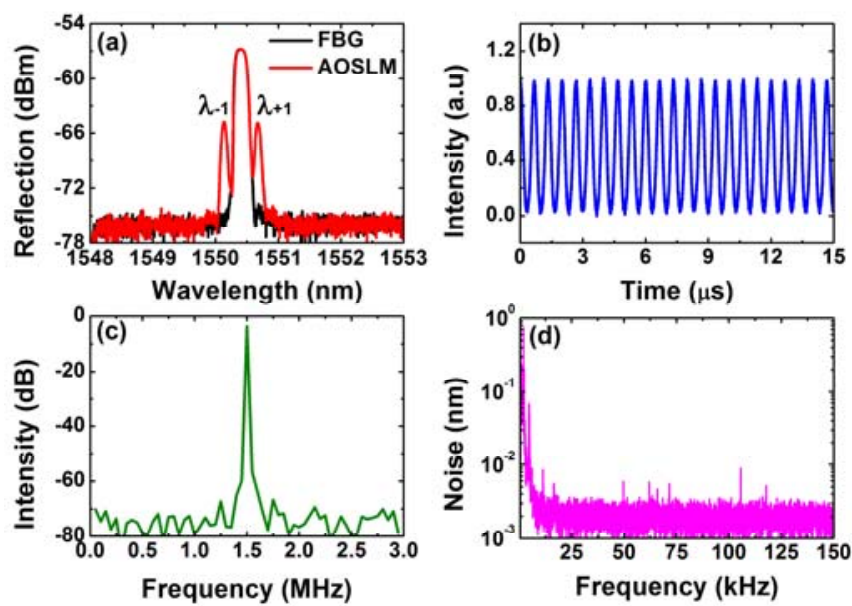

Fig. 2. (a) Reflection spectra of the FBG and the AOSLM structure; (b) Carrier signal of the all-fiber optical heterodyne detection configuration; (c) Fourier frequency spectrum of the carrier signal; (d) Background noise spectrum of the optical heterodyne configuration by demodulating the carrier signal.

Upon measuring the vibration of PZM, a laser beam with a central wavelength equal to that of the +1 -order reflection peak is used to generate the carrier signal without $\mathrm{RF}_{2}$ applied on the PZM. After adjusting the polarizations of the two beams with a polarization controller (PC) in Fig 1, the temporal waveform of the carrier signal is optimized and then recorded with time span of $1 \mathrm{~s}$. However, only a section of carrier signal with time span of $15 \mu$ s is selected to clearly display the visibility of the carrier signal, as shown in Fig. 2(b). Figure 2(c) is the corresponding Fourier spectrum of the carrier signal with a frequency bandwidth of $1 \mathrm{~Hz}$, which shows that the carrier frequency is $1.5 \mathrm{MHz}$ and has an acceptable SNR of $\sim 70 \mathrm{~dB}$. Figure 2(d) shows the background noise spectrum of the optical heterodyne configuration obtained by demodulating the carrier signal. The background noise decreased from $1 \mathrm{~nm}$ to $0.01 \mathrm{~nm}$ with increasing frequency, so as to determine the detectable limit of the all-fiber optical heterodyne configuration.

Furthermore, a sinusoidal signal from $\mathrm{RF}_{2}$ with $f_{0}=60 \mathrm{kHz}$ and $\mathrm{V}_{\mathrm{pp}}=2 \mathrm{~V}$ was applied on the PZM, and then the vibration information was acquired by the carrier signal to generate the phase modulated signal, which was record by an oscilloscope (DPO3054). Figures 3(a) and 3(b) show the temporal waveform of the phase modulated signal and its Fourier spectrum, respectively. From Fig. 3(b), it can be known that there are four new frequency components, $f_{\mathrm{s}}-2 f_{0}, f_{\mathrm{s}}-f_{0}, f_{\mathrm{s}}+f_{0}$ and $f_{\mathrm{s}}+2 f_{0}$, on both sides of the fundamental frequency $f_{\mathrm{s}}$ as predicted according to Eq. (5). The demodulated result (black curve) is shown in Fig. 3(c), which agrees well to the theoretical calculation (red-dash curve) with $U=1.2 \mathrm{~nm}$ and $f_{0}=60 \mathrm{kHz}$. By increasing $\mathrm{V}_{\mathrm{pp}}$ of $\mathrm{RF}_{2}$, the vibration amplitude of the PZM is also measured. The demodulated results are shown in Fig. 3(d). There is good linearity in the relationship between the vibration and the driving voltage with a slope of $0.75 \mathrm{~nm} / \mathrm{V}$. Note that the vibration amplitude increases from $0.013 \mathrm{~nm}$ to $14.8 \mathrm{~nm}$ with $\mathrm{V}_{\mathrm{pp}}$ increasing from $0.4 \mathrm{~V}$ to $20 \mathrm{~V}$. With gradual decrease of $\mathrm{V}_{\mathrm{pp}}$, the vibration information is blended in the background noise. Therefore, the minimum measurable vibration in this optical heterodyne configuration was determined by the background noise and the minimum value was measured to be as small as $0.013 \mathrm{~nm}$. The resolution is around $10 \mathrm{pm}$ due to the controlling voltage accuracy of $0.01 \mathrm{~V}$, which is presented as the pink dots in the inset of Fig. 3(d). Furthermore, we also measured the frequency response of the PZM in the frequency range of $1 \mathrm{~Hz}$ to $150 \mathrm{kHz}$ with $\mathrm{V}_{\mathrm{pp}}=10 \mathrm{~V}$. The vibration amplitude decreased in general with increasing driving frequency. This is similar with the case reported in [14], so the measured results of the frequency response is not presented here.
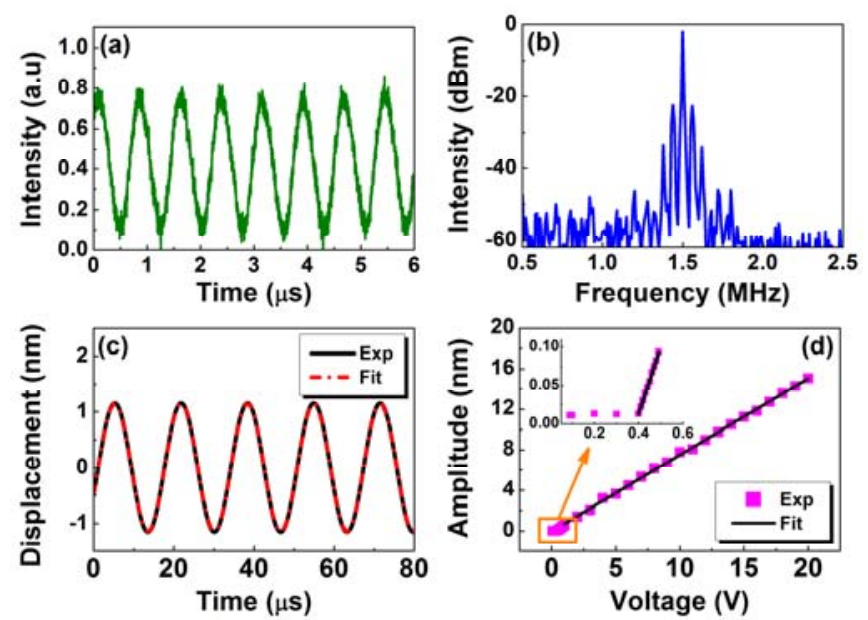

Fig 3. (a) Temporal waveform of the phase modulated signal produced by the all-fiber optical heterodyne detection configuration; (b) Fourier frequency spectrum of the phase modulated signal; (c) Vibration waveform obtained by demodulating the phase modulated signal. The black curve is the demodulated experimental data and the red-dash curve is the theoretical calculation with $U=1.2 \mathrm{~nm}$ and $f_{0}=60 \mathrm{kHz}$; (d) Relationship between the vibration amplitude of PZM and the driving voltage $\mathrm{V}_{\mathrm{pp}}$ with $f_{0}=60 \mathrm{kHz}$.

Apart from the sinusoidal vibration, the measurement of the complicated waveforms and related displacements were also experimentally achieved. As proofs of the principle, the displacements produced by two special waveforms with $f_{0}=1$ $\mathrm{kHz}$ and $\mathrm{V}_{\mathrm{pp}}=5 \mathrm{~V}$ applied on the PZM were measured. The results are shown in Fig. 4, where the demodulated vibration waveforms (blue curves) show good agreement with the normalized waveforms of the driving signals (black curves). 
Furthermore, we make a comparative analysis of the Fourier spectra between the driving signals and the demodulated displacement waveforms, as shown in Figs. 4(c) and 4(d), respectively. Note that the demodulation result has the same spectral component as that of the driving signal, which means that the carrier signal of the optical heterodyne detection configuration can record almost all frequency components of the vibration objects.
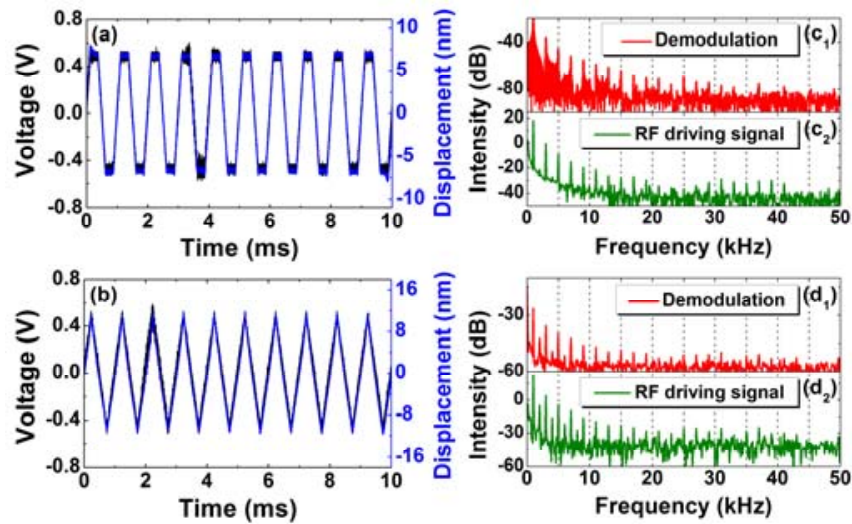

Fig 4. (a) and (b) Two special vibrating waveforms (blue curves) demodulated by the all-fiber optical heterodyne configuration and the corresponding driving voltage waveforms (black curves) applied on the PZM; $\left(\mathrm{c}_{1}\right)$ and $\left(\mathrm{c}_{2}\right)$ Fourier frequency spectra of the trapezoidal wave driving voltage and the demodulated vibrating waveforms; $\left(\mathrm{d}_{1}\right)$ and $\left(\mathrm{d}_{2}\right)$ Fourier frequency spectra of the triangle wave driving voltage and the demodulated vibrating waveforms.

\section{CONCLUSION}

In summary, we demonstrated an all-fiber optical heterodyne micro-vibration detection configuration based on an AOSLM structure. The vibration information of the PZM was loaded onto the carrier signal and a phase modulated signal was generated. The practical vibration information can be obtained by demodulating the phase modulated signal. In our experiment, the vibration waveform within a frequency range between $1 \mathrm{~Hz}$ to $150 \mathrm{kHz}$ was measured by using this optical heterodyne detection configuration. The minimal measurable vibration amplitude and resolution are around $0.013 \mathrm{~nm}$ and $10 \mathrm{pm}$ in a frequency range from tens to hundreds of kilohertz, respectively. This configuration not only has characteristics of compact size, easy-alignment and non-contact measurement, but also gains high measurement accuracy, which could be applied in compact and portable instruments based on all-fiber optical heterodyne detection.

\section{REFERENCES}

11] T. Kessler, C. Hagemann, C. Grebing, T. Legero, U. Sterr, F. Riehle, M. J. Martin, L. Chen, and J. Ye, "A sub-40-mHz-linewidth laser based on a silicon single-crystal optical cavity," Nat. Photon. vol. 6, no. 10, pp. 687-692, 2012.

[2] L. He, Ş. K. Özdemir, J. Zhu, W. Kim, and L. Yang, "Detecting single viruses and nanoparticles using whispering gallery microlasers," Nat. Nanotechnol. vol. 6, no. 7, pp. 428-432, 2011

[3] G. Pernot, M. Stoffel, I. Savic, F. Pezzoli, P. Chen, G. Savelli, A. Jacquot, J. Schumann, U. Denker, I. Mönch, C. Deneke, O. G. Schmidt, J. M. Rampnoux, S. Wang, M.Plissonnier, A. Rastelli, S. Dilhaire, and N. Mingo, "Precise control of thermal conductivity at the nanoscale through individual phonon-scattering barriers," Nat. Mater. vol. 9, no. 6, pp. 491-495, 2010.
[4] S. Blaize, B. Bérenguier, I. Stéfanon, A. Bruyant, G. Lérondel, P. Royer, O. Hugon, O. Jacquin, and E. Lacot, "Phase sensitive optical near-field mapping using frequency-shifted laser optical feedback interferometry," Opt. Express. vol. 16, no. 16, pp. 11718-11726, 2008

[5] C. Moon, S. Lee, and J. K. Chung, "A new fast inchworm type actuator with the robust I/Q heterodyne interferometer feedback," Mechatronics. vol. 16, no. 2, pp. 105-110, 2006.

[6] P. Yang, G. Xing, and L. He, "Calibration of high-frequency hydrophone up to $40 \mathrm{MHz}$ by heterodyne interferometer," Ultrasonics. vol. 54, no. 1, pp. 402-407, 2014

[7] J. B. Abbiss, and W. T. Mayo, "Deviation-free Bragg cell frequency-shifting," Appl. Opt. vol. 20, no. 4, pp. 588-553, 1981.

[8] K. K. Wong, R. M. De La Rue, and S. Wright, "Electro-optic-waveguide frequency translator in $\mathrm{LiNbO}_{3}$ fabricated by proton exchange," Opt. Lett. vol. 7 , no. 11 , pp. 546-548, 1982 .

[9] Y. Li, S. Meersman, and R. Baets, "Realization of fiber-based laser Doppler vibrometer with serrodyne frequency shifting," Appl. Opt. vol. 50, no. 17, pp. 2809-2814, 2011.

[10] Y. Li, S. Verstuyft, G. Yurtsever, S. Keyvaninia, G. Roelkens, D. V. Thourhout, and R. Baets, "Heterodyne laser Doppler vibrometers integrated on silicon-on-insulator based on serrodyne thermo-optic frequency shifters," Appl. Opt. vol. 52, no. 10, pp. 2145-2152, 2013.

[11] T. A. Birks, P. St. J. Russell, and D. O. Culverhouse, "The acousto-optic effect in single-mode fiber tapers and couplers," J. Lightwave Technol. vol. 14, no. 11, pp. 2519-2529, 1996.

[12] B. Y. Kim, H. E. Engan, H. J. Shaw, and J. N. Blake, "All-fiber acousto-optic frequency shifter," Opt. Lett. vol. 11, no. 6, pp. 389-391, 1986.

[13] W. J. Lee, B. K. Kim, K. H. Han, and B. Y. Kim, "Dual heterodyne polarization diversity demodulation for fiber-optic interferometers," IEEE Photon. Technol. Lett. vol. 11, no. 9, pp. 1156-1158, 1999.

[14] W. Zhang, W. Gao, L. Huang, D. Mao, B. Jiang, F. Gao, D. Yang, G. Zhang, J. Xu, and J. Zhao, "Optical heterodyne micro-vibration measurement based on all-fiber acousto-optic frequency shifter," Opt. Express vol. 23, no. 13, pp. 17576-17583, 2015.

[15] P. St. J. Russell, and W. F. Liu, "Acousto-optic superlattice modulation in fiber Bragg gratings," J. Opt. Soc. Am. A vol. 17, no. 8, pp. 1421-1429, 2000.

[16] R. A. Oliveira, J. Canning, K. Cook and A. A. P. Pohl, "Bragg grating writing in acoustically excited optical fiber," Appl. Phy. Lett. vol. 97, no. 4, pp. 041101, 2010.

[17] R. A. Oliveira, C. A. F. Marques, K. Cook, J. Canning, R. N. Nogueira, and A. A. P. Pohl, "Complex Bragg grating writing using direct modulation of the optical fiber with flexural waves," Appl. Phy. Lett. vol. 99 , no. 16, pp. 161111, 2011

[18] R. E. Silva, M. A. R. Franco, P. T. Neves, H. Bartelt, and A. A. P. Pohl, "Detailed analysis of the longitudinal acousto-optical resonances in a fiber Bragg modulator," Opt. Express vol. 21, no. 6, pp. 6997-7007, 2013.

[19] C. Cuadrado-Laborde, A. Díez, J. L. Cruz, and M. V. Andrés, "Doubly active Q switching and mode locking of an all-fiber laser," Opt. Lett. vol. 34 , no. 18 , pp. $2709-2711,2009$

[20] C. Cuadrado-Laborde, A. Díez, M. Delgado-Pinar, J. L. Cruz, and M. V. Andrés, "Mode locking of an all-fiber laser by acousto-optic superlattice modulation," Opt. Lett. vol. 34, no. 7, pp. 1111-1113, 2009.

[21] Y. O. Barmenkov, J. L. Cruz, A. Díez, and M. V. Andrés, "Electrically tunable photonic true-time-delay line," Opt. Express vol. 18, no. 17, pp. 17859-17864, 2010.

[22] W. F. Liu, P. St. J. Russell, and L. Dong, "100\% efficient narrow-band acoustooptic tunable reflector using fiber Bragg grating," J. Lightwave Technol. vol. 16, no. 11, pp. 2006-2009, 1998

[23] T. A. Birks, S. G. Farwell, P. St. J. Russell, and C. N. Pannell, "Four-port fiber frequency shifter with a null taper coupler," Opt. Lett. vol. 19, no. 23, pp. 1964-1966, 1994.

[24] W. Zhang, L. Huang, F. Gao, F. Bo, L. Xuan, G. Zhang, and J. Xu, "Tunable add/drop channel coupler based on an acousto-optic tunable filter and a tapered fiber," Opt. Lett. vol. 37, no. 7, 1241-1243, 2012.

[25] W. Zhang, L. Huang, K. Wei, P. Li, B. Jiang, D. Mao, F. Gao, T. Mei, G. Zhang, and J. Zhao, "High-order optical vortex generation in a few-mode fiber via cascaded acoustically driven vector mode conversion," Opt. Lett. vol. 41, no. 21, pp. 5082-5085, 2016.

[26] W. Zhang, K. Wei, L. Huang, D. Mao, B. Jiang, F. Gao, G. Zhang, T. Mei, and J. Zhao, "Optical vortex generation with wavelength tunability based 
on an acoustically-induced fiber grating," Opt. Express vol. 24, no. 17, pp 19278-19285, 2016.

[27] D. I. Yeom, H. S. Park, and B. Y. Kim, "Tunable narrow-bandwidth optical filter based on acoustically modulated fiber Bragg grating," IEEE Photon. Technol. Lett. vol. 16, no. 5, pp. 1313-1315, 2004.

Authors' biographies not available at the time of publication. 